\section{Is There a Role for LAMP-2 Autoantibodies in Patients with Antineutrophil Cytoplasmic Antibody-associated Vasculitis?}

\section{To the Editor:}

Antineutrophil cytoplasmic antibody (ANCA)-associated vasculitides (AAV) form a group of necrotizing small-vessel vasculitides characterized by the presence of ANCA against either proteinase 3 (PR3) or myeloperoxidase (MPO). ANCA have a key role in the pathogenesis of AAV, inducing excessive activation of neutrophils, which results in injury to small vessels ${ }^{1}$. ANCA can target other neutrophil-derived molecules, among them lysosome-associated membrane glycoprotein 2 (LAMP-2).

LAMP-2 is a glycosylated membrane protein expressed in lysosomes and on the surface of neutrophils and glomerular cells ${ }^{2}$. Antibodies against LAMP-2 were originally detected in cases with active AAV and pauciimmune crescentic glomerulonephritis ${ }^{3}$. Subsequent experimental studies showed that passive immunization with rabbit IgG to recombinant LAMP-2 or active immunization with recombinant FimH (a bacterial adhesion protein present in gram-negative bacteria and sharing 1 epitope of LAMP-2) can induce pauciimmune crescentic glomerulonephritis in rats, thus supporting the pathogenicity of anti-LAMP-2 antibodies ${ }^{4}$.

Patients with active AAV defined as a Birmingham Vasculitis Activity Score (BVAS) of $\geq 3$ were included in our study. Serum concentration of anti-LAMP-2 antibodies was determined using a commercial ELISA kit according to the instructions of manufacturer (PD-H07441, Puda Scientific). Seropositivity for LAMP-2 antibodies was defined as a value higher than the upper reference limit of the control group 5 .

Fifty-nine patients with newly diagnosed or relapsing granulomatosis with polyangiitis (GPA) or microscopic polyangiitis were enrolled. The median BVAS was 16.5 at the time of testing (Table 1). Twenty-three of 35 newly diagnosed patients were sampled 4-6 weeks after initiation of immunosuppressive therapy, whereas 12 were treatment-naive at the time of testing. All patients with relapsing AAV received maintenance therapy. In 28 patients, repeated samples were obtained during stable remission after a median of 16 months. Thirty-six healthy volunteers ( 9 men and 27 women, average age $55.7 \pm 12.4 \mathrm{yrs}$ ) comprised the control group. Using data from the control group, the upper reference level of anti-LAMP-2 antibodies was defined as $48.9 \mathrm{ng} / \mathrm{ml}$. The study was approved by the Sechenov First Moscow University Institutional Review Board (IRB approval number 06-104). All patients gave informed consent for participation in our research study.

In patients with AAV, the median concentration of anti-LAMP-2 antibodies was higher than in the control group: $42.1 \mathrm{ng} / \mathrm{ml}(95 \% \mathrm{CI}$ $39.7-45.9)$ and $37.1 \mathrm{ng} / \mathrm{ml}$ (95\% CI 35.7-39.2), respectively ( $\mathrm{p}<0.001)$. However, only $10.1 \%$ tested positive for these antibodies (Figure 1). Most of them had newly diagnosed AAV with BVAS score of 12 to 26, and were classified as GPA. Of the 6 positive patients, 5 showed only modestly increased LAMP-2 antibodies titers. There was no correlation between the concentration of anti-LAMP-2 antibodies and BVAS score $(r=0.038$, $\mathrm{p}=0.772$ ).

All patients with anti-LAMP-2 antibodies were seropositive for PR3-ANCA. Nevertheless, median concentrations of anti-LAMP-2 antibodies were similar in patients with PR3-ANCA and MPO-ANCA [41.4 $(35.5,43.4)$ and $44.2(38.3,46.1)$, respectively; $\mathrm{p}=0.884]$. Moreover, antiLAMP-2 antibodies were found in only 1 of 12 patients $(8.3 \%)$ with a new diagnosis of AAV and no history of any immunosuppressive treatment. At remission, concentration of anti-LAMP-2 antibodies decreased below reference value in all 6 who were seropositive, whereas it increased slightly above the reference value in 1 seronegative patient. The median concentration of LAMP-2 antibodies did not change following achievement of remission (44.0 and $39.8 \mathrm{ng} / \mathrm{ml} ; \mathrm{p}=0.079$ ).

Our findings suggest that anti-LAMP-2 antibodies are not useful for clinical evaluation of patients with AAV and play a minor role, if any, in disease pathogenesis. On the contrary, Kain, et al detected anti-LAMP-2 antibodies in $80-91 \%$ of 64 patients with newly diagnosed AAV from 3 European centers and showed high concordance $(80.5 \%)$ among the 3 assays
Table 1. Demographic and clinical features of 59 patients with AAV.

\begin{tabular}{lc}
\hline Characteristics & Values \\
\hline Females, $\mathrm{n}(\%)$ & $37(62.7)$ \\
Average age, yrs, mean, \pm SD & $52.5 \pm 14.3$ \\
Diagnosis, n (\%) & \\
GPA & $41(69.5)$ \\
MPA & $18(30.5)$ \\
Newly diagnosed AAV, $\mathrm{n}(\%)$ & $35(59.3)$ \\
Visceral disease, $\mathrm{n}(\%)$ & \\
Pulmonary & $29(49.1)$ \\
Renal & $37(62.7)$ \\
ENT & $38(64.4)$ \\
Eyes & $14(23.7)$ \\
ANCA, n (\%) & \\
PR3-ANCA & $36(61.0)$ \\
MPO-ANCA & $13(22.0)$ \\
Unspecified & $4(6.8)$ \\
Negative & $6(10.2)$ \\
Previous immunosuppression, $\mathrm{n}(\%)$ & $47(79.7)$ \\
Glucocorticoids* & 47 \\
Induction therapy** & 23 \\
Maintenance therapy*** & 24 \\
Laboratory, median $(95 \%$ CI) & \\
Serum creatinine, mmol/1 & $105.2(95.7-200.7)$ \\
ESR, mm/h & $55.0(38.0-64.0)$ \\
CRP, mg/l & $10.2(9.8-36.7)$ \\
Median BVAS (95\% CI) & $16.5(13.6-16.9)$ \\
& \\
&
\end{tabular}

* Median dose of prednisone was $12 \mathrm{mg}(13-22)$. ** Induction therapy: rituximab $(n=4)$, cyclophosphamide $(n=20)$, methotrexate $(n=3)$, or mycophenolate mofetil (MMF; $\mathrm{n}=1)$. *** Maintenance therapy: glucocorticoids only $(\mathrm{n}=12)$, azathioprine $(\mathrm{n}=8)$, methotrexate $(\mathrm{n}=2)$, and MMF $(n=2)$. GPA: granulomatosis with polyangiitis; MPA: microscopic polyangiitis; ANCA: antineutrophil cytoplasmic antibody; AAV: ANCA-associated vasculitis; PR3: proteinase 3; MPO: myeloperoxidase; ESR: erythrocyte sedimentation rate; CRP: C-reactive protein; BVAS: Birmingham Vasculitis Activity Score.

(ELISA, Western blot, and an indirect immunofluorescence assay) ${ }^{6}$. Anti-LAMP-2 antibodies rapidly became undetectable after the initiation of immunosuppressive treatment and frequently became detectable again during clinical relapse ${ }^{6}$. In line with our results, Roth, et al reported anti-LAMP-2 reactivity in $21 \%$ of 329 ANCA-positive patients, in $29 \%$ of 104 ANCA-negative patients, and in $16 \%$ of 104 patients with a FimH-positive urinary tract infection ${ }^{7}$. Anti-LAMP-2 antibodies titers were usually low, and did not correlate to disease activity scores ${ }^{7}$.

Methodologic issues may be responsible for the conflicting results of our and previous studies ${ }^{8}$. Kain and Rees suggested that the existing controversies will be resolved once robust "clinical grade" assays have been developed $^{2}$. Thus far, no such efforts have been undertaken. The results published by Roth and colleagues and ours clearly argue against a role of LAMP-2 in $\mathrm{AAV}^{7}$. The fate of non-reproduced published findings is well established, with confirmation in below $50 \%$ of the scientific reports ${ }^{9}$. This number may be even more pronounced, because reports of positive findings are published more often and more quickly compared to negative data ${ }^{10}$

Our study has some limitations. The number of patients was relatively small. However, we do not expect that our findings would have been different with a larger sample size given the very low prevalence of anti-LAMP-2 reactivity.

SERGEY MOISEEV (D, MD, Tareev Clinic of Internal Diseases, Sechenov First Moscow State Medical University, and Lomonosov Moscow State University; ANASTASIIA ZYKOVA 『, MD, Tareev Clinic of Internal 


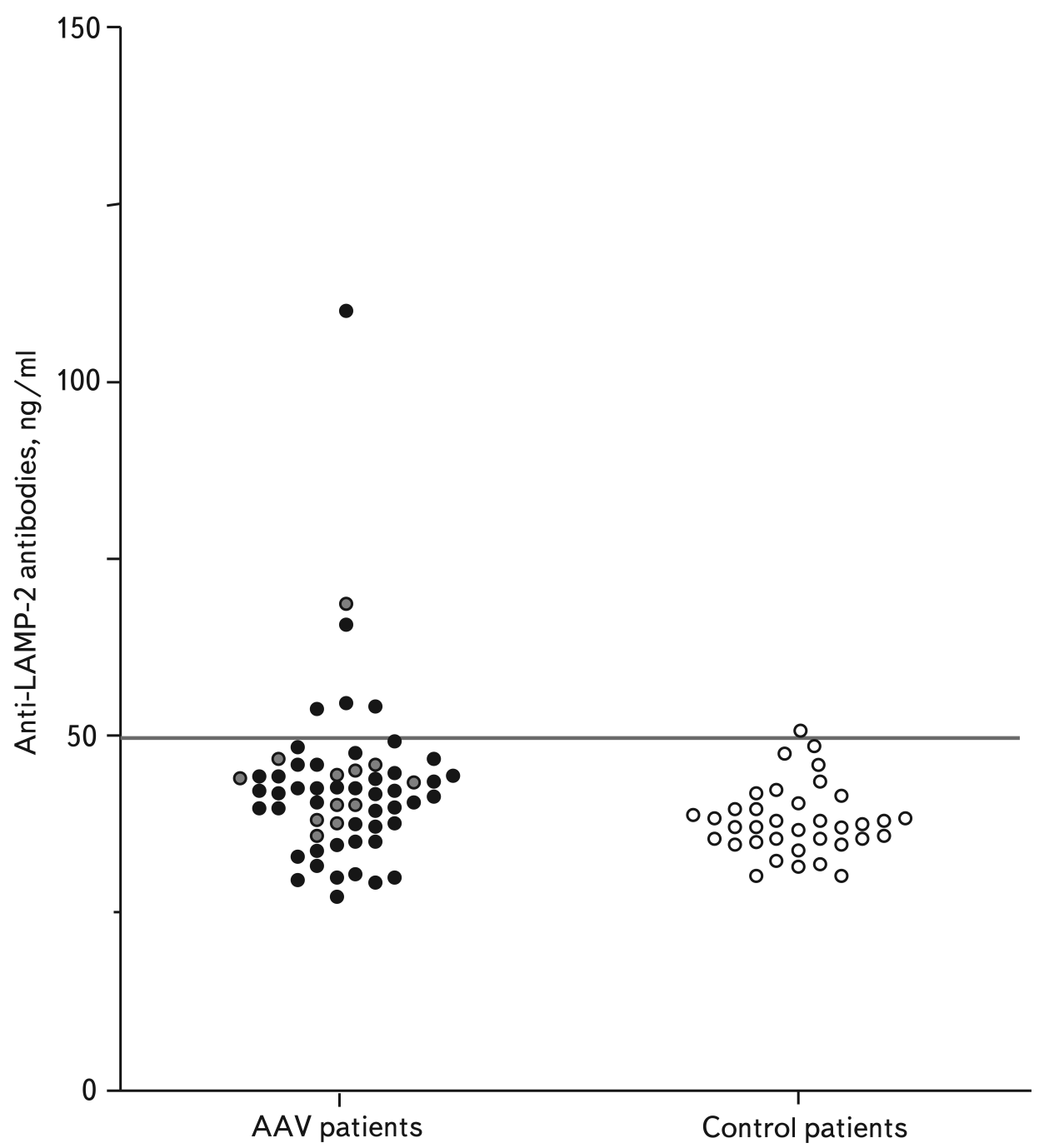

Figure 1. Concentration of anti-LAMP-2 antibodies in patients with AAV and in the control group. Treatment-naive patients marked in gray. LAMP-2: lysosome-associated membrane glycoprotein 2; AAV: antineutrophil cytoplasmic antibody-associated vasculitides.

Diseases, Sechenov First Moscow State Medical University, and Lomonosov Moscow State University, Moscow, Russia; NIKOLAY BULANOV (1), MD, Tareev Clinic of Internal Diseases, Sechenov First Moscow State Medical University; EVGENY GITEL (10, MD, Sechenov First Moscow State Medical University; PAVEL NOVIKOV, MD, Tareev Clinic of Internal Diseases, Sechenov First Moscow State Medical University; MAYRA BULANOVA, MD, Vladimir Regional Clinical Hospital, Vladimir, Russia; ANDREAS KRONBICHLER P , MD, PhD, Department of Internal Medicine IV (Nephrology and Hypertension), Medical University Innsbruck, Innsbruck, Austria; DAVID JAYNE, MD, FRCP, FRCPE, FmedSci, Department of Medicine, University of Cambridge, Cambridge, UK. The study was supported by a grant from Sechenov University. Dr. Kronbichler has received an unrestricted grant from the Austrian Society of Rheumatology. Address correspondence to Dr. S. Moiseev, Tareev Clinic of Internal Diseases, Sechenov First Moscow State Medical University, Rossolimo, 11/5, Moscow, 119435, Russia.

E-mail: avt420034@yahoo.com

\section{REFERENCES}

1. Nakazawa D, Masuda S, Tomaru U, Ishizu A. Pathogenesis and therapeutic interventions for ANCA-associated vasculitis. Nat Rev Rheumatol 2019;15:91-101.

2. Kain R, Rees AJ. What is the evidence for antibodies to LAMP-2 in the pathogenesis of ANCA associated small vessel vasculitis? Curr Opin Rheumatol 2013;25:26-34.

3. Kain R, Matsui K, Exner M, Binder S, Schaffner G, Sommer EM, et al. A novel class of autoantigens of anti-neutrophil cytoplasmic antibodies in necrotizing and crescentic glomerulonephritis: the lysosomal membrane glycoprotein h-lamp-2 in neutrophil granulocytes and a related membrane protein in glomerular endothelial cells. J Exp Med 1995;181:585-97.

4. Kain R, Exner M, Brandes R, Ziebermayr R, Cunningham D, Alderson CA, et al. Molecular mimicry in pauci-immune focal necrotizing glomerulonephritis. Nat Med 2008;14:1088-96.

5. Sasse EA. Determination of reference intervals in the clinical laboratory using the proposed guideline National Committee for Clinical Laboratory Standards C28-P. Arch Pathol Lab Med 
1992;116:710-3.

6. Kain R, Tadema H, McKinney EF, Benharkou A, Brandes R, Peschel A, et al. High prevalence of autoantibodies to hLAMP-2 in anti-neutrophil cytoplasmic antibody-associated vasculitis. J Am Soc Nephrol 2012;23:556-66.

7. Roth AJ, Brown MC, Smith RN, Badhwar AK, Parente O, Chung H, et al. Anti-LAMP-2 antibodies are not prevalent in patients with antineutrophil cytoplasmic autoantibody glomerulonephritis. J Am Soc Nephrol 2012;23:545-55.

8. Fervenza FC, Specks U. Vasculitis: Will LAMP enlighten us about
ANCA-associated vasculitis? Nat Rev Nephrol 2012;8:318-20.

9. Begley CG, Ellis LM. Drug development: raise standards for preclinical cancer research. Nature 2012;483:531-3.

10. Hopewell S, Loudon K, Clarke MJ, Oxman AD, Dickersin K. Publication bias in clinical trials due to statistical significance or direction of trial results. Cochrane Database Syst Rev 2009;(1):MR000006.

First Release February 15 2020; J Rheumatol 2020;47:4; doi:10.3899/jrheum.191082 